\title{
Deuterium NMR Studies on the Orientational Order in Dimeric Thermotropic Liquid Crystals
}

\author{
Hirokazu TORIUMI, Hidemine FURUYA, and Akihiro ABE \\ Department of Polymer Chemistry, Tokyo Institute of Technology, \\ 2-12-1 Ookayama, Meguro-ku, Tokyo 152, Japan
}

(Received February 28, 1985)

\begin{abstract}
KEY WORDS Dimeric Liquid Crystals / Deuterium NMR / Orientational Order Parameter / Odd-Even Effect /
\end{abstract}

There has been an increasing interest focused on thermotropic liquid crystalline polymers comprising a rigid mesogenic core and a flexible spacer group in a repeating unit. $^{1}$ A low molecular weight analog composed of two mesogenic groups interconnected by a flexible spacer chain is often regarded as a prototype of these polymers. ${ }^{2-6}$ A typical example may be found among polyesters of the type

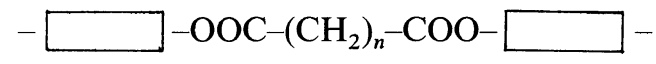

These polymers exhibit an odd-even oscillation in the nematic-isotropic transition temperature when plotted against the number of carbon atoms $(n)$ involved in the spacer. The odd-even effect is even more distinct in the corresponding enthalpy and entropy changes at the transition. Dimer model compounds having analogous flexible spacers are known to exhibit very similar transition behavior. ${ }^{2-6}$

In this paper, we wish to report some preliminary results of our deuterium NMR studies on dimer model compounds of $n=7$ and 8:

bis-4-(4'-methoxyphenyloxycarbonyl)phenyl alkanedicarboxylates

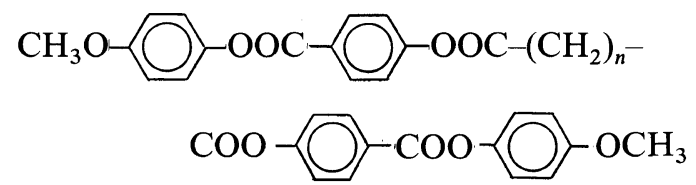

We shall designate these compounds as D7 and D8. They carry partially deuterated phenylene groups at the terminals to provide informations regarding the orientational order parameter of the rigid core in the mesophase. For comparison, the corresponding monomer model compounds are also synthesized:

4-(4'-methoxyphenyloxycarbonyl)phenyl alkanecarboxylates<smiles>CCCCCC(=O)OOc1ccc(C(=O)Oc2ccc(OC)cc2)cc1</smiles>

Notations M3 and M4 will be used to differentiate two samples with $n=3$ and 4 , respectively. All of these compounds exhibit a welldefined nematic mesophase under the microscope. As shown in the due course, samples D7 and D8 show a typical odd-even alteration in their thermodynamic properties.

\section{EXPERIMENTAL}

Deuterium labels were introduced in the terminal phenylene groups by using deuterated p-methoxyphenol as a starting material. Deuteration reaction was carried out by refluxing a mixture of $p$-methoxyphenol and deuterium oxide in the presence of Raney nickel catalyst for one week. This procedure was repeated twice to accomplish $65 \%$ deuterium 
exchange. Details of the synthesis will be reported in a separate communication. ${ }^{7}$

The NMR spectra were recorded on a JEOL JNM-GX-270 Fourier transform spectrometer operating at $41.3 \mathrm{MHz}$ deuterium resonance frequency. Measurements were carried out under a complete proton decoupling, and typically 500 FID signals were accumulated to improve the signal to noise ratio. The liquid crystalline materials were degassed and sealed in conventional $5 \mathrm{~mm}$ NMR tubes. In the measurements, samples initially kept at a temperature above $T_{\mathrm{N}-\mathrm{I}}$ were cooled slowly to turn into the nematic mesophase. During this process, the initial orientation of the sample tube relative to the magnetic field was maintained (non-spinning mode).

\section{RESULTS AND DISCUSSION}

The phase transition temperatures, $T_{\mathrm{K}-\mathrm{N}}$ and $T_{\mathrm{N}-\mathrm{I}}$, determined by DSC are summarized in Table I. The enthalpy changes $\Delta H_{\mathrm{N}-\mathrm{I}}$ associated with the nematic-isotropic transition are also included. Examples of the protondecoupled deuterium NMR spectra are shown for D7 and D8 in Figure 1. Both spectra were obtained in the nematic region, and thus dominated by a symmetric quadrupolar doublet arising from deuterons attached to the phenylene rings. General features of the spectra are the same for the two monomer model compounds (M3 and M4), and there-

Table I. Thermodynamic data of dimeric and monomeric liquid crystals ${ }^{a}$

\begin{tabular}{|c|c|c|c|}
\hline \multirow{2}{*}{ Compounds } & $T_{\mathrm{K}-\mathrm{N}}$ & $T_{\mathrm{N}-\mathrm{I}}$ & $\Delta H_{\mathrm{N}-\mathrm{I}}$ \\
\hline & $\mathrm{K}$ & K & $\mathrm{kcal} \mathrm{mol}^{-1}$ \\
\hline D8 & 384 & 465 & 1.51 \\
\hline D7 & 376 & 456 & 0.61 \\
\hline M4 & 306 & 352 & 0.18 \\
\hline M3 & 304 & 352 & 0.14 \\
\hline
\end{tabular}

a Values are from DSC measurements at a cooling rate of $10 \mathrm{deg} \mathrm{min}^{-1}$ : symbols $\mathrm{K}, \mathrm{N}$, and I represent solid, nematic and isotropic phases, respectively. fore they are omitted. The spacing of the doublet, i.e., the quadrupolar splitting $\Delta v$, provides a measure of the orientational order
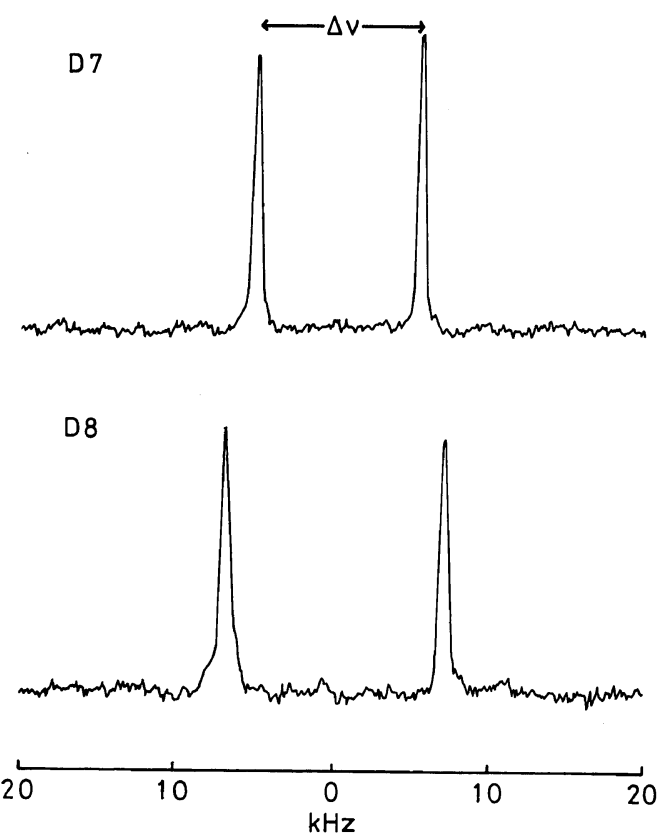

Figure 1. Proton-decoupled deuterium NMR spectra of dimers D7 and D8.

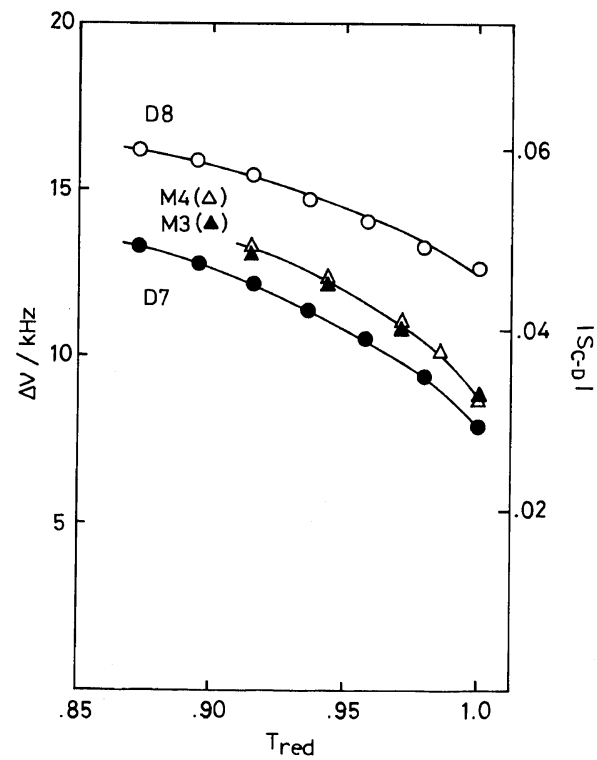

Figure 2. Variation of quadrupolar splitting $\Delta v$ and orientational order parameter of the C-D bond $S_{\mathrm{C}-\mathrm{D}}$ as a function of reduced temperature $T_{\text {red }}:$ D8 $(O), D 7(O)$, M4 $(\triangle)$, and M3 ( $\mathbf{\Delta})$. 
of the C-D bond vector relative to the liquid crystal director:

$$
\Delta v=(3 / 2)\left(e^{2} q Q / h\right) S_{\mathrm{C}-\mathrm{D}}
$$

where the parenthesized quantity, $e^{2} q Q / h$, is called the quadrupolar coupling constant and $S_{\mathrm{C}-\mathrm{D}}$ represents the order parameter. In Figure 2, the observed values of $\Delta v$, and values of $S_{\mathrm{C}-\mathrm{D}}$ calculated therefrom by adopting $e^{2} q Q / h=180 \mathrm{kHz}^{8}$ are plotted as a function of the reduced temperature $T_{\text {red }}\left(=T / T_{\mathrm{N}-\mathrm{I}}\right)$. In all cases, $\Delta v$ values decrease gradually as the temperature increases. The difference in $\Delta v$, and thus in $S_{\mathrm{C}-\mathrm{D}}$, is strikingly large between the two dimers. The corresponding values for the monomers are nearly identical with each other and located in the intermediate region. It should be noted here that the order parameter of the ring para axis $\left(S_{\mathrm{Zz}}\right)$ may not be directly deduced from these $S_{\mathrm{C}-\mathrm{D}}$ values in the case when the mesogenic core is of low symmetry (less than $\mathrm{C}_{3}$ ) and its ordering tensor involves non-zero biaxiality. Such phenomena are often encountered in various monomeric liquid crystals. ${ }^{9}$ In the present study, we have carried out a reference experiment by using a dimer model compound which bears the $\mathrm{CD}_{3}-$ groups at the terminals instead of $\mathrm{CH}_{3} \mathrm{O}$ - of D8. This compound exhibits well-resolved quadrupolar and dipolar splittings, leading to an unambiguous determination of the tensor elements. ${ }^{10}$ In consideration of these results, we assume that the terminal methoxyphenyl groups of the present compounds would possess the biaxiality identical to that of the methylphenyl group at a given $T_{\text {red }}$. The $S_{\mathrm{Zz}}$ values thus estimated at $T_{\mathrm{N}-\mathrm{I}}$ are as follows: 0.60 (D8), 0.38 (D7), 0.42 (M4), and 0.43 (M3).

These results immediately indicate that the orientational order of the mesogenic core is very much different between dimers carrying even- and odd-membered flexible spacer chains. For a dimer with $n=$ even, the colinear alignment of the two terminal mesogenic cores is one of the preferred conformations, and thus it would facilitate molecular packing with high order of orientation. In the case of $n=$ odd, however, the parallel alignment of the terminal cores should require high-energy conformations of the spacer chain. Such a disparity should be responsible for the odd-even oscillation observed in various thermodynamic quantities at the nematic-isotropic transition. ${ }^{11}$

We summarize the enthalpy changes $\Delta H_{\mathrm{N}-\mathrm{I}}$ determined at $T_{\mathrm{N}-1}$ in the fourth column of Table I. According to the theory, ${ }^{12,13}$ the latent heat of the transition is given as a function of the square of the order parameter, the axial ratio of the mesogenic group, and a mean interaction parameter. Since compounds D7 and D8 are homologous in their chemical constitution, the observed difference in $\Delta H_{\mathrm{N}-\mathrm{I}}$ should arise mainly from the orientation term, the contribution from the latter two factors being negligibly small. In the present examples, the ratio $\Delta H_{\mathrm{N}-\mathrm{l}} / S_{\mathrm{Zz}}{ }^{2}$ is calculated to be nearly identical for the two dimers ( $c a$. 4.2). For a more quantitative discussion, further investigations are in progress.

Acknowledgements. We are grateful to $\mathrm{Mr}$. K. Kosaka and Mr. N. Fujii of JEOL for measuring deuterium NMR spectra. Financial support from Asahi Glass Foundation for Industrial Technology is also acknowledged.

\section{REFERENCES}

1. "Polymer Liquid Crystals," A. Ciferri, W. R. Krigbaum, and R. B. Meyer, Eds., Academic Press, New York, N.Y., 1982.

2. J.-I. Jin, Y.-S. Chung, R. W. Lenz, and C. Ober, Bull. Korean Chem. Soc., 4, 143 (1983); J.-I. Jin and J.-H. Park, Mol. Cryst. Liq. Cryst., 110, 293 (1984).

3. J. W. Emsley, G. R. Luckhurst, G. N. Shilstone, and I. Sage, Mol. Cryst. Liq. Cryst., 102, 223 (1984).

4. J. A. Buglione, A. Roviello, and A. Sirigu, Mol. Cryst. Liq. Cryst., 106, 169 (1984).

5. G. Sigaud, D. Y. Yoon, and A. C. Griffin, Macromolecules, 16, 875 (1983).

6. R. B. Blumstein, M. D. Poliks, E. M. Stickles, A. Blumstein, and F. Volino, Mol. Cryst. Liq. Cryst., submitted.

7. H. Furuya, H. Toriumi, and A. Abe, to be submitted. 
8. R. G. Barnes and J. W. Bloom, J. Chem. Phys., 57, 3082 (1972).

9. See, for example, R. Y. Dong, E. Tomchuk, C. G. Wade, J. J. Visintainer, and E. Bock, J. Chem. Phys., 66, 4121 (1977); J. M. Emsley, S. K. Khoo, and G. R. Luckhurst, Mol. Phys., 37, 959 (1979); J. W. Emsley, G. R. Luckhurst, and C. P. Stockley, Mol. Phys., 44,
565 (1981).

10. H. Toriumi, H. Furuya, and A. Abe, in preparation.

11. A. Abe, Macromolecules, 17, 2280 (1984).

12. W. Maier and A. Sauper, Z. Naturforsch., 14a, 882 (1959); 15a, 287 (1960).

13. P. J. Flory and G. Ronca, Mol. Crystl. Liq. Cryst., 54, 311 (1979). 